\title{
Vibration-Based Indoor Human Sensing Quality Reinforcement via Thompson Sampling
}

\author{
Tong $\mathrm{Yu}$ \\ Carnegie Mellon University \\ Pittsburgh, Pennsylvania, USA \\ worktongyu@gmail.com
}

\author{
Yue Zhang \\ University of California Merced \\ Merced, California, USA \\ yzhang58@ucmerced.edu
}

\author{
Zhizhang $\mathrm{Hu}$ \\ University of California Merced \\ Merced, California, USA \\ zhu42@ucmerced.edu
}

\author{
Susu Xu \\ Stony Brook University \\ Stony Brook, New York, USA \\ susu.xu@stonybrook.edu
}

\author{
Shijia Pan \\ University of California Merced \\ Merced, California, USA \\ span24@ucmerced.edu
}

\begin{abstract}
This paper presents an online learning algorithm that recommends sensor locations for structural vibration-based indoor human sensing systems to achieve optimal sensing quality for high learning accuracy. The intuition is that we model the deployment environment features (physics models) to reflect the environmental impact on the signals, which would further impact the sensing applications' performance. We acquire these features by re-purposing the ambient sensing data used for sensing applications. Then we combine these environment features and the application performance acquired as user feedback (data-driven knowledge) to recommend the sensor locations with optimal sensing quality. We formulate the task as a multi-armed bandit problem and develop an online learning algorithm based on Thompson sampling. Real-world datasets are collected to validate the proposed algorithm for both online and offline learning scenarios. The system achieves 56\% for R@1 and $90 \%$ for $R @ 3$, which demonstrate an up to $2.5 \times$ improvement compared to baseline approaches.
\end{abstract}

\section{CCS CONCEPTS}

- Computer systems organization $\rightarrow$ Sensor networks; $\bullet$ Computing methodologies $\rightarrow$ Model development and analysis.

\section{KEYWORDS}

Sensing quality, online learning, structural vibration sensing, indoor human monitoring

\section{ACM Reference Format:}

Tong Yu, Yue Zhang, Zhizhang Hu, Susu Xu, and Shijia Pan. 2021. VibrationBased Indoor Human Sensing Quality Reinforcement via Thompson Sampling. In The First International Workshop on Cyber-Physical-Human System Design and Implementation (CPHS21), May 18, 2021, Nashville, TN, USA. ACM, New York, NY, USA, 6 pages. https://doi.org/10.1145/3458648.3460012

Permission to make digital or hard copies of all or part of this work for personal or classroom use is granted without fee provided that copies are not made or distributed for profit or commercial advantage and that copies bear this notice and the full citation on the first page. Copyrights for components of this work owned by others than ACM must be honored. Abstracting with credit is permitted. To copy otherwise, or republish, to post on servers or to redistribute to lists, requires prior specific permission and/or a fee. Request permissions from permissions@acm.org.

CPHS21, May 18, 2021, Nashville, TN, USA

(C) 2021 Association for Computing Machinery.

ACM ISBN 978-1-4503-8440-7/21/05 . .\$15.00

https://doi.org/10.1145/3458648.3460012

\section{INTRODUCTION}

With the rapid growth of Cyber-Physical Systems (CPS) and Internetof-Things (IoT), more and more smart home devices with the ability of sensing indoor human information have entered people's life. It is estimated that by the year 2023 there will be close to 2.7 billion installed smart home devices in the United States [1]. These smart home devices rely on various sensors to capture indoor human information and provide autonomous services accordingly. We focus on structural-vibration based human sensing systems due to its non-intrusiveness for long-term fine-grained indoor human sensing $[8,10]$. Despite of these advantages, this 'developing' sensing modality is prone to environment variance - the human information learning accuracy varies over different deployment environments and sensor placements. This variance in system performance directly impacts the quality of user experience and limits the applicability of the system.

Prior work on structural vibration-based human sensing demonstrates that the deployment environment directly impacts the quality of data acquisition (sensing) procedure and the system's human information inference accuracy $[17,28]$. Even within the same building/area, different sensor locations may result in a wide range of performance variance due to heterogeneity of structural layout, materials, and structural elements (e.g., beams and partitions) [6]. Quantifying these impacts provides a systematic way to evaluate and understand the limitation of sensing systems' sensing procedure [28]. On the other hand, these structural vibration-based sensing devices are (semi-)mobile, i.e., the sensor is placed on the monitored surface [18], and can be moved. In addition, when installed on in-home mobile platforms, such as a robot vacuum cleaner, it provides fine-grained options of sensor locations [9]. As a result, updating sensor location may enhance the structural vibration sensing quality and in turn improve the system information inference accuracy.

In this work, we explore online learning approaches to provide sensor location recommendations to enhance the deployment sensing quality and learning accuracy. Our algorithm takes into account both the understanding of the deployment environment features (physics models) that may impact the sensing quality and the performance feedback (data-driven knowledge) into account for the agent to provide recommendation of sensor locations. Therefore, the contributions of this work are as follows 
- We present an online reinforcement learning based scheme to enhance vibration-based human sensing deployment's data acquisition quality.

- We utilize the acquired human-induced sensing signal for both application information inference and deployment environment modeling.

- We utilize environmental modeling (physics models) and user feedback (data-driven knowledge) to achieve online recommendation for sensor placement and improve system accuracy.

- We collect real-world data over different deployments and sensor placements to validate our proposed scheme.

\section{MOTIVATION}

The performance of device-free human sensing systems is often impacted by deployment environment features. Taking structural vibration based human sensing as an example, the sensing task performance may be impacted by various environment features such as decay model, structural material homogeneity, structural elements [6, 28]. To demonstrate the significance of the problem, we deployed sensors for the pedestrian identification application over multiple environments. In each environment, we investigate four difference sensor placements. Figure 1 demonstrates system performance in the form of identification accuracy over three sensing areas (different building or floor) when sensors are placed at different locations (noted as 1-4). We observe that for the same system deployed at different sensing areas to sense the same set of target, the information inference accuracies vary. This variance can be as high as $20 \%$. In addition, for the same sensing area, different sensor locations also result in information inference accuracy variance. For example, at the Sensing Area 1, the average learning accuracy variance over different sensor locations may vary up to $10 \%$. These inference accuracy variance could be induced by the structural variance such as decay model, dominant structural mode, etc. With the uncertainty of estimating the accuracy (i.e., variance), it is non-trivial to select the best location simply based on the observed accuracy in all candidate locations. In the above example, in the Sensing Area 2, sometimes a higher accuracy will be reported in Location 1 while sometimes higher accuracy will be reported in Location 3. With this uncertainty, it is difficult to determine whether Location 1 is better than Location 3, or not. Therefore, it is desired to handle the uncertainty by learning the system over time in an online fashion. By developing algorithms to efficiently acquire data and user feedback online, we can reduce the uncertainty over time and achieve better understanding of which location leads to better sensing quality. To develop efficient algorithms, we need to balance between optimizing the sensing quality based on the knowledge already acquired (i.e., exploitation) and exploring new locations to further acquire knowledge (i.e., exploration).

\section{SYSTEM AND ALGORITHM DESIGN}

The system mainly consists of four modules, sensing module, human information inference (e.g., pedestrian/activity recognition), deployment environment modeling, and online learning for sensor placement recommendation. As shown in Figure 2, the sensing module captures human induced vibration (Section 3.1), the data is

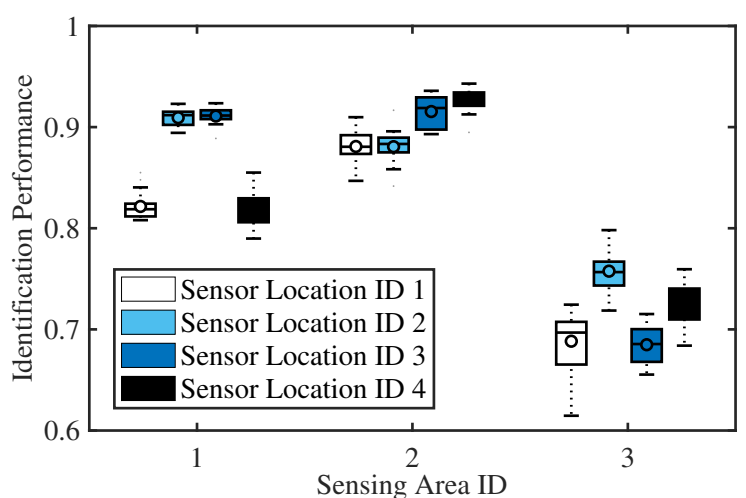

Figure 1: Motivation. The same sensing system performance vary over different sensing area as well as different sensor locations.

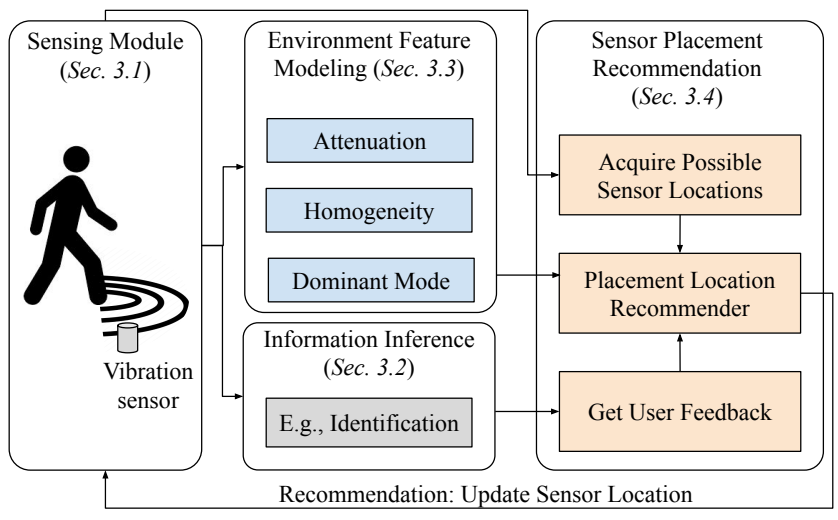

Figure 2: System Overview.

sent to both information inference module for application (Section 3.2), as well as environment feature modeling module to extract deployment sensing quality indicators (Section 3.3). The environment features and the user feedback (application performance indicator) are then used by the placement location recommender to provide sensor location update recommendation.

\subsection{Sensing System Design and Configuration}

The structural vibration-based indoor human sensing system utilizes vibration sensors, SM-24 geophone, to capture human-induced structural vibration to further infer occupant information [19]. The intuition is that when people interact with structures, e.g., footfall, touch, knock, these interactions excite the structure. The generated vibration propagates through the structure and reaches the vibration sensor mounted on the corresponding surface. Because human-induced vibration is of low voltage, we use an operational amplifier to amplify the sensor signal before digitizing it [20]. An Arduino node with Analog-to-Digital (ADC) modules is used to digitize and process the signal. These signals are then sent to the information inference module for further information extraction. 


\subsection{Information Inference: Pedestrian Identification}

Human-induced structural vibration can be used for various human information inference, such as identity, location, activity, gait parameters, etc. The intuition is that people interact with the ambient environment and induce the vibrations of surfaces around them. These vibration signals can be captured by structural vibrations sensors and used to infer the signal source - human information.

We focus on the application of pedestrian identification as an example because it is essential information needed for various smart building applications. The footstep-induced vibration signal segment, which is referred to as a Step Event (SE), is first extracted through an anomaly detection algorithm [19]. Frequency domain features are extracted from the detected signal segments. We form the identification problem as a binary classification problem (with two occupants), where a Support Vector Machine (SVM) with linear kernel is used to identify the pedestrian based on the features extracted from their footstep-induced floor vibration signals [19].

\subsection{Environment Feature Modeling}

Our system further uses the acquired human-induced vibration signal to model features of the deployed sensor location. For each sensor location, we extract $D=5$ number of features to represent deployment properties, which are listed below.

- Attenuation Model: we extract the decay rate $(\alpha)$ as well as the amplitude $\left(A m p_{0}\right)$

- Homogeneity Model: we calculate the similarity of the same excitation over different propagation path $(\mathrm{H})$

- Dominant Vibration Mode Characteristics: where the widths of the dominant frequency are calculated (DFW1, DFW2)

The attenuation model reflects the signal strength distribution over the target sensing area. To estimate the attenuation model parameters, we utilize the relative locations of the detected footstepinduced vibration signals and the measured footstep signal energy at these relative locations. We consider the mechanical wave attenuation model [24] as a function of sensor-footstep distance $d$ $A m p(d)=\frac{A m p_{0}}{\sqrt{d}} e^{-2 \alpha d}$ where $\alpha$ is the decay rate and $A m p_{0}$ is the initial amplitude of the vibration wave created by an excitation before attenuation by propagation. When a person walks by the sensor, their SE signal energy increases when they approach and decreases when they leave. Figure 3 demonstrates relative locations between the sensor and footsteps when a person walks by. We consider the SE with the highest signal energy (usually the step that is the closest to the sensor, e.g., S3) as the reference $S E$ that is $d_{\text {ref }}$ away from the sensor. We then estimate the rest of SE locations accordingly assuming an approximate footstep length of $l$. For an $\mathrm{SE}$ that is $k$ step away from the reference SE, we consider its distance to the vibration sensor $d_{k}$ calculated as: $d_{k}=\sqrt{d_{r e f}^{2}+(k l)^{2}}$. In practice, we assume $d_{r e f}=2 \mathrm{ft}$ and $l=2 \mathrm{ft}$. We use all the SEs collected in each deployment to fitting the attenuation model and estimate $\alpha$ and $A m p_{0}$.

The homogeneity of the deployment determines the data distribution shift over different propagation paths of the area. To measure the homogeneity of the area where the sensor is located, we pair the SEs from the same walking trace that have the same distance

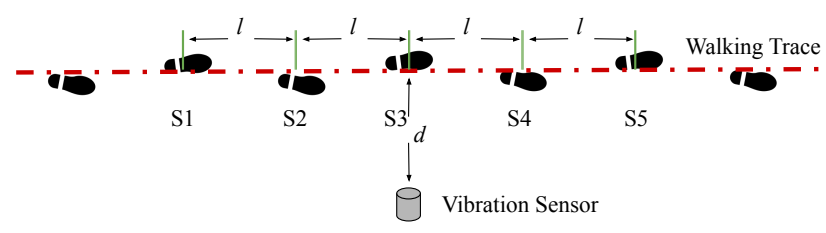

Figure 3: The relative location relationship between the sensor and the footsteps when the participant walking through. $S 1$ to $S 5$ are the footstep-induced vibration signals that belong to a same trace.

from the sensor, and measures the maximum value of the cross correlation of the two pairwise SEs. As demonstrated in Figure 3, the pairwise SEs that have the same distance from the reference SE are $(\mathrm{S} 1, \mathrm{~S} 5)$ and $(\mathrm{S} 2, \mathrm{~S} 4)$. For each sensor placement at each deployment, we estimate this environment feature as the maximum value of all the pairwise SE similarity from all walking traces.

The dominant vibration mode reflects the frequency response characteristics of the excitation, i.e., the efficiency of the frequency response to reflect excitation difference. When different types of excitation only triggers a single structure's dominant vibration mode, most of the signal energy concentrates on a narrow frequency band and makes the signals difficult to be distinguished. We calculate DFW1 and DFW2 with the reference SE in each walking trace, and report the average value over all walking traces for each location.

We represent deployment environment features of a location by

$$
\mathbf{x}=\left[x^{(1)}, x^{(2)}, \ldots, x^{(5)}\right]
$$

where $x^{(1)}, x^{(2)} x^{(3)}, x^{(4)}$ and $x^{(5)}$ are the values of $\alpha, A m p_{0}, \mathrm{H}$, DFW1 and DFW2 respectively.

\subsection{Online Learning for Sensor Location Recommendation}

We develop an online learning algorithm to recommend the deployment sensor locations that allow high-fidelity data acquisition, such that the system can achieve the optimized performance (i.e., accuracy). To effectively leverage the deployment environment features, we use a linear Thompson sampling algorithm, which learns the model $\theta$ on the sensor location's environment feature $\mathbf{x}$.

At each time, the learning agent interacts with the real world as follows. First, the agent receives possible sensor locations (provided by the CPS/IoT sensing system) and deployment environment features at each sensor location. Then, by the model, the agent recommends the best sensor location from the possible locations. Finally, the users provides the feedback, and the recommender updates the model accordingly. We formulate this task as a multi-armed bandit problem, where each possible sensor location corresponds to an arm and the user feedback is used as the reward. We further develop an online learning algorithm based on the Thompson Sampling to maintain and operate on the model, considering its strong empirical performance [4] compared to other bandit algorithms.

We consider at time $t$, the location selected by our learning agent has environment features as $\mathbf{x}_{t} \in \mathbb{R}^{1 \times D}$ (detailed in Section 3.3) and the user feedback received is $y_{t}$. In practice, the feedback is developed as the accuracy of the system during the time $t$, which 
is a continuous variable and with uncertainty (detailed in Section 2). To model this uncertainty, we assume that the user feedback of location $\mathrm{x}_{t}$ at time $t$ follows a normal distribution

$$
y_{t} \sim \mathcal{N}\left(\mathbf{x}_{t} \theta^{*}, \sigma^{2}\right),
$$

where the vector $\theta^{*} \in \mathbb{R}^{D \times 1}$ represents $D$ unknown bandit model parameters. We further assume that the conjugate prior on $\theta^{*}$ also follows a normal distribution

$$
\mathcal{N}\left(\mathbf{0}, \lambda^{-1} I_{D}\right),
$$

where the mean is 0 and the covariance matrix is $\lambda^{-1} I_{D}$.

At time step $t+1$, our algorithm works as follows. First, the bandit model parameters are sampled from its posterior. We denote $X_{t}=\left(\mathbf{x}_{1}^{\top}, \ldots, \mathbf{x}_{t}^{\top}\right)^{\top}$ as a $t \times D$ matrix of the features of all selected locations in $t$ steps and $Y_{t}=\left(y_{1}, \ldots, y_{t}\right)^{\mathrm{T}} \in \mathbb{R}^{t \times 1}$ as the received rewards in $t$ steps. By Bayes' theorem, the posterior at time $t$ is sampled from a normal distribution

$$
\theta_{t+1} \sim \mathcal{N}\left(\bar{\theta}_{t}, S_{t}\right),
$$

where the mean is $\bar{\theta}_{t}=\sigma^{-2} S_{t} X_{t}^{\mathrm{\top}} Y_{t}$ and the covariance matrix is $S_{t}=$ $\left(\sigma^{-2} X_{t}^{\top} X_{t}+\lambda I_{D}\right)^{-1}$. Second, the system selects and recommends the location with the highest expected reward by

$$
\mathbf{x}_{t+1}=\arg \max _{\mathbf{x} \in \text { all locations at } t+1} \mathbf{x} \theta_{t+1} .
$$

Then, the system receives reward $y_{t+1}$ derived from the system performance, which in our case is the pedestrian identification accuracy (Section 3.2). Based on this reward, we further update the posterior of the bandit model for the next time step.

\section{EVALUATION}

To evaluate the proposed scheme, we collect real-world data with aforementioned hardware and users. In this section, we will first introduce the data collection procedure. Next, we explain the baseline approaches used and experimental settings. Finally, we demonstrate results and provide analysis.

\subsection{Data Collection}

We collected real-world pedestrian footstep-induced floor vibration signals from multiple deployment environments. We deployed four vibration sensors that contain the same version of hardware modules and with the same configuration in each environment. We ask the participant to walk by the sensors multiple times with varying distances to the sensors. We collect the floor vibration signals when the pedestrian walks by in their natural gait with two participants. Then we conduct both the pedestrian identification (application) and the environment feature estimation with this data. To ensure the robustness of the system, we collected the data when each participant wears different types of shoes. For each scenario uniquely identified by < pedestrian, shoe type, environment $>$, we collect six traces where the pedestrian passes all sensor locations, resulting in a total of over 250 SEs for each participant.

\subsection{Sensor Location Recommendation Baselines}

We compare our algorithm to several baselines: random selection, greedy and $\epsilon$-greedy [25]. By random selection, the agent will randomly select one location at each step, ignoring the feature of locations. This baseline represents user randomly selecting the sensor location in real world sensing task practise. By greedy, at time $t$ the agent will pick one location with the highest average accuracy estimated on the observations from time 1 to time $t-1$. The greedy algorithm wants to choose the action with the best estimate at each time step, yet provides no efficient way to form these estimates. It purely exploits the information available, but does none of the exploration required to generate this information. By $\epsilon$-greedy, there is an probability value of $\epsilon \in[0,1]$ to control the agent to explore the set of all possible actions. In other words, the agent will behave as greedy algorithm $1-\epsilon$ of the time and will explore random alternatives $\epsilon$ of the time.

\subsection{Experiment Settings}

We evaluate our system and algorithm in both online setting and offline setting. To get averaged results, in total we run the experiment for 7 times. In each experiment, we learn the model in randomly selected 5 environments. In online setting we evaluate the model in the selected 5 environments, while in the offline setting we evaluate the model in the other 2 holdout environments.

In the online setting, we assume the users visit the 5 environments sequentially over time. In this setting, we evaluate the cumulative accuracy (i.e., reward) of the algorithms in the 5 environments over time. Specifically, the average cumulative reward at time $n$ is $\frac{1}{n} \sum_{t=1}^{n} r_{t}$, where $r_{t}$ is the reward received at time $t$. This metric has been widely used to reflect the cumulative performance of online learning and reinforcement learning algorithms [3, 4, 22]. With the randomness, we run each online experiment for 200 runs. With 7 times of experiments, the average results for $7 \times 200=1400$ runs with standard errors are reported.

In the offline setting, we assume the users visit the 5 environments sequentially over time. After the model learns for $T=$ $20,50,75,100$ time steps, we evaluate the system in the other 2 unknown and different environments. We evaluate the performance under Recall@k [23, 27]. For each environment, the agent is provided with 4 candidate locations. The goal of the agent is to rank the locations by assigning a higher score to the location where the sensing system has better quality, and assigning lower scores to the location where the sensing system has lower quality. Under the metric of Recall@k, the prediction result is correct if the best location is among the top $\mathrm{k}$ selected locations from the 4 candidate locations. The average recall results over the 7 times of experiments are reported.

\subsection{Results and Analysis}

4.4.1 Online Evaluation. We evaluate how our system learns over time in the online setting. We compare the average cumulative reward of different algorithms in Figure 4. There are several observations. First, we observe a clear gap between greedy and $\epsilon$ greedy. The improvement by $\epsilon$-greedy validates the importance of introducing exploration to handle the uncertainty when estimating the accuracy of the sensing system. Second, we observe that the performance of $\epsilon$-greedy varies significantly with different $\epsilon$. To achieve high accuracy by $\epsilon$-greedy, a careful tuning of $\epsilon$ is needed. We try $\epsilon=\{0.1,0.2, \ldots, 0.9\}$ and report the best performance of 
Table 1: Evaluation in the offline setting. $R @ k$ is short for Recall@k.

\begin{tabular}{|c|c|c|c|c|c|c|c|c|c|c|c|c|}
\hline & \multicolumn{3}{|c|}{$\mathrm{T}=20$} & \multicolumn{3}{|c|}{$\mathrm{T}=50$} & \multicolumn{3}{|c|}{$\mathrm{T}=75$} & \multicolumn{3}{|c|}{$\mathrm{T}=100$} \\
\hline & R@1 & R@2 & R@3 & R@1 & R@2 & R@3 & R@1 & R@2 & R@3 & R@1 & R@2 & R@3 \\
\hline Thompson Sampling & $47.89 \%$ & $57.71 \%$ & $91.82 \%$ & $53.79 \%$ & $59.21 \%$ & $90.79 \%$ & $55.11 \%$ & $60.25 \%$ & $90.04 \%$ & $56.07 \%$ & $60.43 \%$ & $90.14 \%$ \\
\hline Random Selection & $25.29 \%$ & $48.89 \%$ & $74.43 \%$ & $24.43 \%$ & $50.36 \%$ & $75.25 \%$ & $25.79 \%$ & $49.71 \%$ & $75.29 \%$ & $24.39 \%$ & $49.90 \%$ & $74.71 \%$ \\
\hline
\end{tabular}

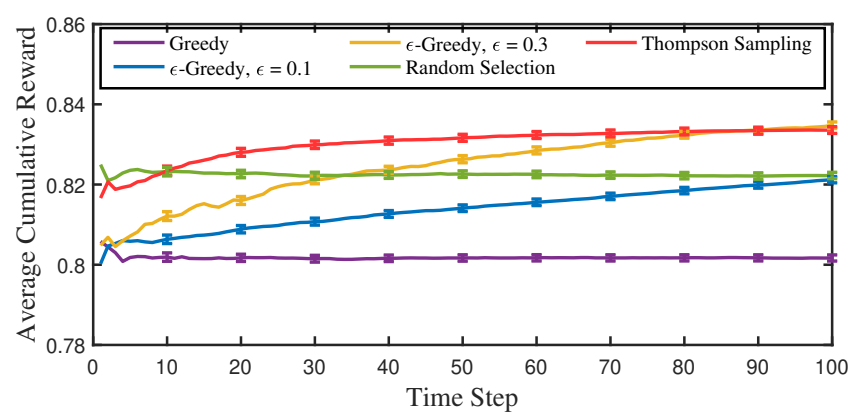

Figure 4: Evaluation in the online setting.

$\epsilon$-greedy, when $\epsilon=0.3$. Instead, Thompson sampling has less hyperparameters to tune. Third, Thompson sampling outperforms all the baselines and achieves steady accuracy increase, especially during the early time steps. The accuracy increase during early time steps is very critical, since the user may lose the interests of providing feedback if their feedback can not effectively improve the system especially in the early time steps. The improvement by Thompson sampling is due to (i) its better exploration and exploitation strategy without tuning the hyper-parameter $\epsilon$ [4] and (ii) its learning of a parameterization on the locations features with a better generalization ability.

4.4.2 Offline Evaluation. To further validate the generalization ability of our system, we evaluate our system in the offline setting. We compare Thompson sampling to the random selection method. The greedy and $\epsilon$-greedy method can not be directly applied to predict in the unknown environment in the offline evaluation, since they do not learn the parameterization on the location features. We only consider $T \leq 100$ considering in the real-world deployment user feedback may be limited.

The Recall@k performances of different algorithms with $\mathrm{T}=20$, 50, 75 and 100 are reported in Table 1 . Taking $\mathrm{T}=100$ as an example, our proposed method achieves a 56.07\% Recall@1 and 60.43\% Recall@2, which means that with a probability of $56.07 \%$, our method can directly find the best location and with a probability of $60.43 \%$ the best location is in top 2 positions of the rank. The probability of selecting the worst location is $1-90.14 \%=9.86 \%$. On the contrary, randomly selecting the location can only achieve 24.39\% Recall@1, 49.90\% Recall@2, and 74.71\% Recall@3. The probability of selecting the worst location is $1-74.71 \%=25.29 \%$, which is $2.5 \times$ higher than the proposed approach. These results validate that randomly placing vibration sensors has a lower chance to achieve the best sensing application performance.

\section{RELATED WORK}

\subsection{Sensing System Quality Assessment}

Prior work has been done on evaluate the quality of cyber-physical sensing systems in terms of signal and data quality [11-13, 15, $26,28]$. For example, signal quality is measured as the property of acquired signal to assess the system performance, such as Carrier-toNoise Ratio (CNR) [11], and structural-similarity index (SSIM) [15, 26]. The data quality is measured as the quality of the existing dataset and further infer the system quality [13]. Karkouch et al. define the IoT data quality with a multi-dimensional definition including accuracy, confidence, completeness, data volume, and timeliness [12]. Zhang et al. measures the sensing signal quality as a series of metrics that describes the deployment's properties and used a data-driven model to predict the system performance with measured properties [28]. In this work, we explored how the quantified environment features, e.g., sensing system quality, can be used to enable optimal sensor location recommendation.

\subsection{Multi-Armed Bandits and Thompson Sampling}

A multi-armed bandit $[3,14]$ is a popular framework for learning to act under uncertainty and handle the exploration and exploitation problem. At each step, the algorithm chooses an arm (i.e., takes an action) and observes a reward from the environment. Based on the reward, the algorithm adapts the strategy of choosing the arm in the next step. The goal of the algorithm to maximize the reward over time. The framework has been successfully applied to many sequential decision making problems, ranging from recommender system, computational advertising, hyper-parameter optimization to reinforcement learning $[4,5,16,22]$.

Several multi-armed bandit algorithms have been proposed with varying strategies of choosing the arm, such as $\epsilon$-greedy, UCB and Thompson sampling [3, 4, 25]. In this paper, for sensor placement, we develop algorithm based on Thompson sampling, considering its strong performance over $\epsilon$-greedy and UCB [4]. The real-world problem is very complex, with various underlying structures. To model the varying underlying structures and achieve efficient learning, existing works have proposed different parameterizations of the reward value, such as linear functions, polynomial functions and neural networks $[2,7,21]$.

\section{DISCUSSION}

We further discuss the limitation and future work in this section. In this paper, we use the system performance of the application as the user feedback. In the real-world deployment, the user feedback may not be as consistent as in our controlled experiments, and there might be errors and bias induced by human variance. Since the user feedback is limited and it may take a long time to acquire sufficient 
user feedback, the model trained for the sensing application may change over time, which may or may not be impact by the environment features. I.e., the optimal sensor location may vary over time as well. In our future work, we plan to conduct more uncontrolled experiment to test the online learning performance and enhance the robustness of the system/algorithm in practice.

In addition, we implemented the application with training and testing data from each sensor location. In the real-world deployment, there might be even constraints for labeling data, where only initial training sensor locations have the manual labels. Especially for the structural vibration sensing, which is sensitive to the structural layout and materials, this data distribution change will directly impact the learning accuracy [17]. When that happens, not only the system needs to discover new optimal sensor location, it will need to assess the transferbility (transfer learning efficiency/accuracy) of the data collected at new sensor locations. This two tasks need to be done simultaneously with a larger number of possible locations.

\section{CONCLUSION}

In this paper, we presented an online learning algorithm to recommend sensor locations in a target deployment environment for structural vibration based indoor human sensing. We combines the physical knowledge (environment features) and the data-driven knowledge (application performance such as user feedback) to learn the recommendation via a linear Thompson sampling algorithm. Real-world dataset is collected to validate the system and algorithm design. Our system achieves an Recall@1 of 56\% and an Recall@3 of $90 \%$ when used to recommend sensor location with the optimal sensing quality (the highest learning accuracy) for structural vibration based human sensing applications.

\section{ACKNOWLEDGEMENTS}

This research was supported by a 2020 Seed Fund Award from CITRIS and the Banatao Institute at the University of California.

\section{REFERENCES}

[1] [n.d.]. Installed base of smart home devices in U.S. 2018-2023. https://www.statista.com/statistics/1075749/united-states-installed-baseof-smart-home-systems/. Accessed: 2021-03-12.

[2] Yasin Abbasi-Yadkori, Dávid Pál, and Csaba Szepesvári. 2011. Improved Algorithms for Linear Stochastic Bandits.

[3] Peter Auer, Nicolo Cesa-Bianchi, and Paul Fischer. 2002. Finite-time Analysis of the Multiarmed Bandit Problem. Machine Learning 47 (2002), 235-256.

[4] Olivier Chapelle and Lihong Li. 2011. An empirical evaluation of thompson sampling. In Advances in neural information processing systems. 2249-2257.

[5] Wei Chu, Lihong Li, Lev Reyzin, and Robert Schapire. 2011. Contextual bandits with linear payoff functions. In Proceedings of the Fourteenth International Conference on Artificial Intelligence and Statistics. JMLR Workshop and Conference Proceedings, 208-214.

[6] Lixing He, Carlos Ruiz, Mostafa Mirshekari, and Shijia Pan. 2020. SCSV2: physicsinformed self-configuration sensing through vision and vibration context modeling. In Adjunct Proceedings of the 2020 ACM International Joint Conference on Pervasive and Ubiquitous Computing and Proceedings of the 2020 ACM International Symposium on Wearable Computers. 532-537.

[7] Daniel N Hill, Houssam Nassif, Yi Liu, Anand Iyer, and SVN Vishwanathan 2017. An efficient bandit algorithm for realtime multivariate optimization. In Proceedings of the 23rd ACM SIGKDD International Conference on Knowledge Discovery and Data Mining. 1813-1821.

[8] Zhizhang Hu, Tong Yu, Yue Zhang, and Shijia Pan. 2020. Fine-grained activities recognition with coarse-grained labeled multi-modal data. In Adjunct Proceedings of the 2020 ACM International foint Conference on Pervasive and Ubiquitous Computing and Proceedings of the 2020 ACM International Symposium on Wearable Computers. 644-649.
[9] Zhizhang Hu, Yue Zhang, and Shijia Pan. 2021. Poster Abstract: Vibration-based Indoor Occupant Gait Monitoring with Robot Vacuum Cleaners. In Proceedings of International Conference on Internet-of-Things Design and Implementation(IoTDI '21).

[10] Yandao Huang and Kaishun Wu. 2020. Vibration-based pervasive computing and intelligent sensing. CCF Transactions on Pervasive Computing and Interaction (2020), 1-21.

[11] AKM Najmul Islam, Elena Simona Lohan, and Markku Renfors. 2008. Moment based CNR estimators for BOC/BPSK modulated signal for Galileo/GPS. In 2008 5th Workshop on Positioning, Navigation and Communication. IEEE, 129-136.

[12] Aimad Karkouch, Hajar Mousannif, Hassan Al Moatassime, and Thomas Noel. 2016. Data quality in internet of things: A state-of-the-art survey. Fournal of Network and Computer Applications 73 (2016), 57-81.

[13] Anja Klein and Wolfgang Lehner. 2009. Representing data quality in sensor data streaming environments. Fournal of Data and Information Quality (FDIQ) 1, 2 (2009), 1-28.

[14] T. L. Lai and Herbert Robbins. 1985. Asymptotically Efficient Adaptive Allocation Rules. Advances in Applied Mathematics 6, 1 (1985), 4-22.

[15] Chaofeng Li and Alan C Bovik. 2009. Three-component weighted structural similarity index. In Image quality and system performance VI, Vol. 7242. International Society for Optics and Photonics, 72420 Q.

[16] Lisha Li, Kevin Jamieson, Giulia DeSalvo, Afshin Rostamizadeh, and Ameet Talwalkar. 2017. Hyperband: A novel bandit-based approach to hyperparameter optimization. The fournal of Machine Learning Research 18, 1 (2017), 6765-6816.

[17] Mostafa Mirshekari, Jonathon Fagert, Shijia Pan, Pei Zhang, and Hae Young Noh. 2020. Step-Level Occupant Detection across Different Structures through Footstep-Induced Floor Vibration Using Model Transfer. Fournal of Engineering Mechanics 146, 3 (2020), 04019137.

[18] Shijia Pan, Amelie Bonde, Jie Jing, Lin Zhang, Pei Zhang, and Hae Young Noh. 2014. Boes: building occupancy estimation system using sparse ambient vibration monitoring. In Sensors and Smart Structures Technologies for Civil, Mechanical, and Aerospace Systems 2014, Vol. 9061. International Society for Optics and Photonics.

[19] Shijia Pan, Ningning Wang, Yuqiu Qian, Irem Velibeyoglu, Hae Young Noh, and Pei Zhang. 2015. Indoor person identification through footstep induced structural vibration. In Proceedings of the 16th International Workshop on Mobile Computing Systems and Applications. 81-86.

[20] Shijia Pan, Susu Xu, Mostafa Mirshekari, Pei Zhang, and Hae Young Noh. 2017. Collaboratively adaptive vibration sensing system for high-fidelity monitoring of structural responses induced by pedestrians. Frontiers in Built Environment 3 (2017), 28.

[21] Carlos Riquelme, George Tucker, and Jasper Snoek. 2018. Deep Bayesian Bandits Showdown: An Empirical Comparison of Bayesian Deep Networks for Thompson Sampling. In International Conference on Learning Representations.

[22] Daniel Russo, Benjamin Van Roy, Abbas Kazerouni, Ian Osband, and Zheng Wen. 2017. A tutorial on thompson sampling. arXiv preprint arXiv:1707.02038 (2017).

[23] Otmane Sakhi, Stephen Bonner, David Rohde, and Flavian Vasile. 2020. BLOB: A Probabilistic Model for Recommendation that Combines Organic and Bandit Signals. In Proceedings of the 26th ACM SIGKDD International Conference on Knowledge Discovery \& Data Mining. 783-793.

[24] SA Shapiro and G1 Kneib. 1993. Seismic attenuation by scattering: theory and numerical results. Geophysical fournal International 114, 2 (1993), 373-391.

[25] Richard S Sutton and Andrew G Barto. 2018. Reinforcement learning: An introduction. MIT press.

[26] Zhou Wang and Alan C Bovik. 2002. A universal image quality index. IEEE signal processing letters 9, 3 (2002), 81-84.

[27] Nirandika Wanigasekara, Yuxuan Liang, Siong Thye Goh, Ye Liu, Joseph Jay Williams, and David S Rosenblum. 2019. Learning Multi-Objective Rewards and User Utility Function in Contextual Bandits for Personalized Ranking.. In IFCAI. 3835-3841.

[28] Yue Zhang, Lin Zhang, Hae Young Noh, Pei Zhang, and Shijia Pan. 2019. A signal quality assessment metrics for vibration-based human sensing data acquisition. In Proceedings of the 2nd Workshop on Data Acquisition To Analysis. 29-33. 